\title{
La regulación emocional como herramienta efectiva para el mejoramiento pedagógico en la enseñanza de inglés
}

\section{Emotional regulation as an effective tool for pedagogical improvement in the teaching of English}

DOI: $10.46981 /$ sfjhv2n3-003

Received in: April 1st, 2021

Accepted in: May 31th, 2021

\author{
Vivian Coromoto Rojas Ceballos \\ Universidad Estatal de Milagro. \\ Milagro, Ecuador. \\ E-mail:vrojasc@unemi.edu.ec \\ Leslie Jetzabel Folleco Calixto \\ Universidad Estatal de Milagro. \\ Milagro, Ecuador. \\ E-mail: lfollecoc@unemi.edu.ec \\ Wilson Alexander Zambrano Vélez \\ Universidad Estatal Península \\ de Santa Elena. \\ Manabí, Ecuador. \\ E-mail: alexzambrano1@outlook.cl
}

\begin{abstract}
RESUMEN
Este articulo está enmarcado en la necesidad de mejorar las prácticas educativas universitarias hacia la formación integral del alumno, permitiéndole integrar con éxito en el mundo laboral con las suficientes habilidades emocionales y sociales. Para ello, se buscó ayuda metodológica para los docentes de inglés de manera de enfrentar el alto nivel de ansiedad, inseguridad, frustración y temor de los estudiantes de la carrera pedagogía de lenguas nacionales y extranjeros modalidad online de la Universidad Estatal de Milagro. Se realizó un plan de acción basado en estrategias de análisis socioemocional; considerando que las universidades deben superar las barreras que separan la lógica del sentimiento, afectando la interrelación con los estudiantes y su aprendizaje, por lo que debe haber un auto reconocimiento crítico y consciente del porqué del accionar para la comprensión y acción mediante una dialógica multidimensional, generando espacios pedagógicos.
\end{abstract}

Palabras clave: Regulación, emociones, enseñanza, aprendizaje.

\begin{abstract}
This article is framed in the need to improve university educational practices towards the integral formation of the student, allowing them to successfully integrate into the work world with sufficient emotional and social skills. For this, methodological help was sought for English teachers in order to face the high level of anxiety, insecurity, frustration and fear of the students of the online modality Pedagogy of national and foreign languages ty career of the State University of Milagro. An action plan based on socio-emotional analysis strategies was carried out; considering that universities must overcome the barriers that separate logic from feeling, affecting the interrelation with students and their
\end{abstract}


learning, so there must be a critical and conscious self-recognition of the reason to act for understanding and action through a multidimensional dialogic, generating pedagogical spaces.

Keyword: Regulation, emotions, teaching, learning.

\section{INTRODUCCIÓN}

Es claro que se vive inmerso en una era liderada por la indiferencia en todas sus presentaciones, donde la afectividad, la conciencia y la comprensión parecieran no tener cabida. Es lamentable, pero la educación tampoco es la excepción; un caso específico son las clases de inglés. Muchos de los profesores de lenguas extranjeras continúan con la heredada educación colonizada de la falta de motivación para emprender nuevos métodos de enseñanza, para que la práctica deje de ser un proceso mecanizado y rígido. No existe interés ni empatía hacia las demás personas, pareciera que la convivencia estuviera en peligro de extinción. La falta de técnicas humanísticas, hace de la práctica docente, una mera instrucción, donde los estudiantes no son tratados como participantes, sino como números, y sólo se estaría prestando atención a lo intelectual, pero ¿qué ocurre con lo emocional?; sin este factor muy difícilmente se podrá lograr la formación integral del estudiante. La socialización con sus aspectos emocionales, no se desarrolla del todo en el entorno escolar, porque el alumno permanece en las aulas gran parte de su infancia y adolescencia, afrontando cantidad de problemas que se presentan en el terreno educativo tales como fracaso escolar, desmotivación, entre otros; obstaculizando la convivencia social, trayendo como consecuencia la deserción. El caso es, que esta situación no queda solo en los niveles de primaria y segundaria, sino que trasciende a la educación superior, en un efecto cíclico que se duplica. En ese sentido, la universidad separa la lógica del sentimiento y no enseña a vivir en sociedad, pero la sociedad tampoco enseña a estar en contacto con las emociones, y mucho menos a expresarlas.

Siendo las cosas así, al enfrentarse a esta realidad, el aprendizaje de un segundo idioma puede entonces también en ocasiones ser una experiencia traumática para muchos estudiantes, revelando que la emoción más común percibida en este contexto es la ansiedad, afectando también la calidad de la comunicación al bloquearse.

El objetivo consiste en mejorar las prácticas educativas universitarias hacia la formación integral del alumno, permitiéndole integrarse con éxito en el mundo laboral con las suficientes habilidades emocionales y sociales; por lo que se recoge y sistematiza la información de contenido teórico y empírico acerca de las emociones y el mejoramiento pedagógico de una segunda lengua.

En este artículo se realizará una metodología 'de investigación acción de tipo descriptiva reflejando las incidencias en notas de campo a través de la observación, cuestionarios y entrevistas, analizando su contenido para la reflexión y teorización. Posteriormente, se presentarán los resultados 
para su discusión y se elaborarán las conclusiones, que invitarán a continuar la investigación sobre el tema. Por último, se detallará las referencias bibliográficas utilizadas.

\section{DESARROLLO}

\subsection{LA REGULACIÓN EMOCIONAL CONSISTE EN LA HABILIDAD DE GUIAR LAS} EMOCIONES DE MANERA ADECUADA.

Supone tomar conciencia de la relación entre emoción, cognición y comportamiento; tener buenas estrategias de afrontamiento; así como capacidad para autogenerar emociones positivas, ya que la regulación emocional impacta a la motivación. En tal sentido, la familia constituye la base donde irá desarrollándose la regulación emocional [1].

Es importante entonces destacar cómo las competencias sociales de los niños están influidas por el hecho de cómo las madres transmiten sentimientos positivos o negativos en el hogar e incluso antes, en la etapa intrauterina, la cual guarda relación con el tipo de apego y el modo de crianza con el que cuenta una persona, pues la capacidad de regular las emociones de las figuras cuidadoras primarias o padres, incide directamente en cómo los niños y niñas aprenden a autorregularse emocionalmente. Es por esta razón, que la regulación emocional tiene impacto desde los primeros años de vida, logrando un funcionamiento óptimo en la edad adulta [2].

Ante esto, se dice que al menos hay seis factores que potencialmente pueden generar interferencias al aprender una lengua extranjera. Estos factores son los siguientes: La ansiedad personal e interpersonal. Estas actitudes la desencadenan el estudiante, en la falta de un buen concepto de sí mismo, en la materia y posee una baja autoestima al compararse con los demás. Otro factor, son las creencias erróneas o falsas expectativas de los estudiantes sobre el aprendizaje de un idioma. Por ejemplo, si piensan que van a hablar como un nativo en unos pocos meses, terminarán frustrándose. Se dice que "cuando las creencias y la realidad se enfrentan, la ansiedad aparece". El siguiente factor, son las creencias del profesor sobre la enseñanza de un idioma, es decir, profesores que piensan que su función es corregir permanentemente a los alumnos, impartir clases magistrales o ser autoritarios, provocando desmotivación y ansiedad entre los alumnos, afectando la efectiva interacción o relación entre el profesor y los alumnos. La corrección de algunos errores es necesaria, pero lo más importante es la forma como el profesor lo hace, al no ridiculizar al alumno delante del resto de la clase [3].

En el mismo orden de ideas, otro factor, son los métodos utilizados en clase. La principal tarea que causa ansiedad entre los estudiantes es el hablar en público y más aún en otro idioma. Las personas que les crea ansiedad hablar en público son "personas que se preocupan mucho de la evaluación que los demás hacen de ellas" lo cual va de la mano con su autoestima. Esto se ve reflejado en aquellos alumnos que no hablan ni quieren participar, salvo cuando es estrictamente necesario, por no pasar vergüenza, 
producto a que la habilidad oral no fue lo suficientemente promovida en los primeros niveles de sus estudios, sin contar, que pueda estar relacionada con las posibles experiencias no gratas que el estudiante experimentó y vivió en el pasado en su aprendizaje del idioma inglés. Finalmente, los exámenes, los cuales, por norma general, suelen provocar estrés, debido a que se está haciendo una valoración no sólo del rendimiento del alumno, sino también de sus cualidades personales. Ante esto, se ha demostrado que los factores afectivos no operan independientes de los otros, sino que todos están relacionados.

Desde diferentes modelos metodológicos han constatado las carencias en cuanto a inteligencia emocional en la población universitaria. Se dice que, si no se interviene de forma explícita en la formación emocional de los alumnos, sus competencias en este sentido no mejorarán a lo largo de la formación universitaria [4].

De manera pues, un docente que refleje carencias en las competencias emocionales tiende a percibir lo negativo de la realidad y el enjuiciamiento en el aula, donde es necesaria una intervención durante la formación profesional. Investigaciones realizadas muestran que los docentes son cada vez más conscientes de la necesidad de incluir la educación emocional en las aulas y, sin embargo, no han recibido la formación necesaria para desarrollar esta tarea con éxito, desempeñando su labor con aquellos niveles de competencia emocional que han podido desarrollar de una forma casi intuitiva a lo largo de su proceso de socialización [5]

A partir de esto, resulta legítimo cuestionarse ¿cuántos de los profesionales en formación aprenden a conocer, regular y gestionar su propia emocionalidad? y ¿cuántos son capaces de comprender los estados de ánimo de los demás y de enfocar sus actuaciones en función de estrategias asertivas de relación? El logro de estas habilidades emocionales constituye uno de los grandes desafíos de la educación de estos tiempos. Siendo las cosas así, la actitud en el estudiante a veces no es suficiente para dirigir su motivación hacia el estudio, ya que influyen principalmente las emociones que experimenta en el contexto académico. Factores como la autoconfianza y un bajo nivel de ansiedad repercuten directamente en el proceso de aprendizaje [6].

Esto implica, la acción de filtro afectivo la cual radica en que cuando éste es fuerte, el alumno reduce sus posibilidades de obtener un input del idioma comprensible, pues los efectos negativos de las emociones que comprenden el filtro afectivo hacen al mismo estudiante, reducir el contacto que se pueda tener con la lengua que se quiera aprender; o también no penetrar hasta aquellas zonas de la mente que facilitan la adquisición del idioma. Esto basado en que el aprendizaje no conduce al aprendiz a un discurso fluido, ya que las personas están más preocupadas por la forma del idioma, que con lo que vaya a ser transmitido, provocando un bloqueo al estar constantemente pendiente de la producción del idioma, evitándose la espontaneidad en el discurso. Es decir, si no se interviene de forma explícita en la formación emocional de los alumnos, sus competencias en este sentido no mejorarán a lo largo de la 
formación universitaria y lo que es peor, se duplicarán porque lo harán cuando sean docentes con sus futuros estudiantes [7].

Un estudio confirma que prácticamente uno de cada dos alumnos, presenta un déficit en competencias emocionales que incluyen habilidades personales e interpersonales, lo que lleva en un $43,6 \%$ a tener miedo a hablar en público, en un $40 \%$ a no sentirse capaz de liderar un grupo y en un $60,9 \%$ a no intentar asumir el mando e imponer un ritmo de trabajo cuando trabaja en equipo, pudiendo ser quienes ni siquiera logren finalizar la carrera o que arrastren múltiples problemas laborales, puesto que presentan una baja capacidad de automotivación, considerando aspectos como infravalorar el propio trabajo cuando se trabaja en grupo, percibirse como incapaces ante nuevas situaciones y desanimarse ante las dificultades [8].

Un trabajo de investigación basado en la formación del profesorado en educación emocional: Diseño, aplicación y evaluación". considera el papel del docente y cómo las emociones tanto propias como de los estudiantes, deben ser tomadas en cuenta en el acto pedagógico, lo cual sirvió para poner de manifiesto la excelente satisfacción del profesorado en su aplicabilidad y su impacto en la mejora de las relaciones con los estudiantes, permitiendo que haya mayor sensibilización, implicación y motivación para trabajar la educación emocional en las aulas [9].

Asimismo, se presenta otra investigación que proporcionan pautas para el aprendizaje del idioma inglés en la educación superior intercultural, desde una perspectiva conceptual sobre las actitudes, emociones en el ámbito de educativo y su relación con aspectos cognitivos, afectivos, conativos así como comportamental. , donde existe una gran responsabilidad por parte del estudiantado y más aún por el profesorado, en términos de cultivar actitudes y emociones en el proceso de aprendizaje de la competencia comunicativa del idioma inglés, además de incorporar tecnologías de la información y comunicación para incrementar el agrado, la motivación así como la confianza hacia el aprender y comprender inglés [10].

De igual manera, se menciona el siguiente trabajo investigativo basado en la inteligencia emocional en el estudiante universitario de licenciatura durante el proceso de aprendizaje del idioma Inglés, arrojando que la mayoría de los estudiantes universitarios experimentan con mayor frecuencia durante el proceso de aprendizaje del idioma inglés ansiedad, rabia y culpa; por lo que hay que concientizar al docente de las emociones que el estudiante experimenta durante su aprendizaje, brindando un ambiente favorable en clase para mejorar el aprendizaje del idioma inglés [11].

Por último, se muestra una investigación, basado en la inteligencia emocional del docente y la satisfacción académica del estudiante universitario, el cual señala la importancia de la inteligencia emocional del docente en el proceso educativo y la interacción docente-estudiante. Las habilidades emocionales, específicamente las interpersonales, permiten al docente construir y mantener en el tiempo 
relaciones de entendimiento mutuo con los estudiantes, pertenecer al grupo y demostrar cooperación por el bien de todos, comprender y apreciar las emociones y sentimientos, comprometerse con el desempeño académico de los estudiantes y crear ambientes adecuados para el aprendizaje y la satisfacción académica. Se podría decir que el desarrollo de habilidades emocionales en el docente se traduce en indicadores positivos de la satisfacción académica [12].

Ante lo antes expuesto, el lenguaje se considera como la herramienta psicológica más importante, donde se conoce, desarrolla y comprende la realidad del entorno, mediante actividades ricas en contenido afectivo y emocional, mostrando de esta manera satisfacción y motivación en la realización de las mismas, donde se sientan seguros de ocupar un espacio de manera interactiva [13].

A medida que en la persona satisface su impulso de cooperación social, establece relaciones significativas con otras personas y amplía su mundo. Es decir, establece conexiones significativas con una realidad externa componente esencial de la autorrealización, donde no hay cabida al egoísmo y la competencia, las cuales generan emociones hostiles, limita sus relaciones con la realidad externa y su conciencia permanece internamente limitada [14].

En ese sentido, las emociones se construyen a través de un conjunto de procesos neurofisiológicos que requieren una conceptualización compartida socialmente. Es decir, se recibe información sensorial que se transforma en patrones neuronales. El cerebro categoriza las sensaciones y las dota de significado a través de la experiencia previa y del contexto social. En otras palabras, construyen las emociones con una perspectiva diferente para mirar el aprendizaje más allá del aula, invitando a los educadores a reinventarse como tales para dar mejor respuesta a los desafíos que enfrenta la educación, en el presente siglo, desde la perspectiva del conocimiento en acción [15].

\section{METODOLOGÍA}

La metodología empleada en el presente artículo es la investigación acción participativa, que se define como un estudio de una situación social con el fin de mejorar la calidad de la acción dentro de la misma, las cuales van encaminadas mediante la reflexión a modificar la situación. Una vez que se logra una comprensión más profunda de los problemas, lleva hacia interpretaciones para la producción de la teorización sobre la realidad estudiada, con el fin de alcanzar una transformación social [16].

Se implementa el uso de un cuestionario abierto donde se pudo conocer las emociones que experimentan en el proceso enseñanza aprendizaje del idioma inglés, los docentes y estudiantes para comprender su significado. Para el análisis de los datos se implementa la triangulación temporal la cual recoge información de la investigación. Se realizó la categorización, contrastando los tres diferentes momentos, describiendo detalladamente el antes, donde se sitúa la iniciativa, observaciones de la actitud de los docentes y estudiantes; el durante, donde se escalonan las fases de acción con estrategias 
pedagógicas basada en las emociones y el después, donde se demuestra los resultados de la realidad estudiada [17].Se presenta el plan de acción el cual permitirá orientar las ideas de cambio dirigidas a mejorar la realidad existente. Ver tabla 1.

\section{RESULTADOS}

\section{A. Antes}

Se pudo constatar en el estudio realizado, el predominio de los estudiantes hacia la ansiedad, temor y frustración ante el aprendizaje del idioma inglés, mientras que los docentes se mostraron más académicos e investigadores y no acompañantes del desarrollo integral del estudiante. Esto trajo como consecuencia que se aislaran y trabajaran solos. Es pertinente mencionar, que en las clases manifestaron no dejarse ver por temor al docente, les preguntara algo. Preferían no conectarse y ver la clase grabada cuando les tocaba un profesor aburrido o que les genere estrés, enfocado en los errores que los estudiantes puedan cometer, más que al mensaje que se está transmitiendo, siendo distantes, perdiéndose la naturalidad del discurso y del aprendizaje e incidiendo directamente en el aprendizaje del idioma, provocando en el estudiante bloqueo, rechazo o deserción.

\section{B. Durante}

Se mostraron receptivos en la aplicación de estrategias para el manejo de las emociones, promoviendo la consciencia y empatía hacia el estado emocional tanto a docentes como a estudiantes. Se incluyeron estrategias de regulación emocional cognitivas, dirigidas a la regulación de emociones negativas como la aceptación al poder reflexionar y admitir su error, verlo como parte del aprendizaje, un ensayo hacia el éxito y no como un fracaso a través de la revaluación positiva, es decir, ver el lado positivo de las cosas negativas, sacarle provecho al error y seguir trabajando con esfuerzo. Así mismo, se presenta la alteridad, colocarse en el puesto del otro, aprender a escuchar y comprender en lugar de crear supuestos, lejos de la realidad. Por último, las estrategias grupales como apoyo social, para construir soluciones, realizando actividades gratificantes, donde tengan la posibilidad de crear.

\section{Después}

Los docentes, tomando en cuenta estas emociones, al planear las clases, adoptaron una mejor actitud para moderar y manejar la enseñanza del idioma inglés, haciendo sentir cómodo, así como confidente al estudiante con su aprendizaje en cualquier actividad que se lleve a cabo en la clase de inglés. Durante las observaciones realizadas, se pudo evidenciar importantes interacciones, que condujeron al logro de la participación y rapport entre los protagonistas de la investigación, así como el uso de estrategias didácticas como el podcast y los info videos, los cuales ayudaron a mejorar las habilidades orales, auditivas, el vocabulario y la pronunciación, haciendo un discurso más fluido al trabajar en equipo, donde aprendieron los unos con los otros. 


\section{CONCLUSIONES}

\section{A. Emocional}

- Realizar sesiones de realimentación al final de la clase, en donde los estudiantes puedan expresar sus emociones, conocerlas y ayudarles a sobrellevarlas, especialmente cuando experimentan ansiedad, decepción, frustración y enojo mediante el empleo de frases positivas.

- Percibir y comprender el cómo se sienten los estudiantes durante su aprendizaje, orientando al estudiante a descubrir mediante preguntas y situaciones reales, siendo esto atractivo y útil para su futuro.

B. Intelectual

- Empleo de estrategias lúdicas como la dramatización, música, mapas mentales y gamificación, buenos para la convivencia.

- Promover la auto reflexión en clase, capaz de monitorizar su aprendizaje, de auto corregirse, de saber cuándo incurrió en un error, tomar decisiones y actuar.

C. Actitudinal.

- Cuanto más abiertos se encuentren para percibir y reconocer las emociones, mayor habilidad tendrán para percibir y comprender a los demás.

- Ser capaces de identificar los sentimientos en sí mismos y en las personas con las que interactúan, de modo de evitar conflictos y gestionar de forma adecuada la comunicación de sus sentimientos en sus relaciones con los demás.

Tabla 1. Actividades realizadas en el plan de acción.
Actividades
Indicadores
Recursos

\begin{tabular}{llll}
\hline $\begin{array}{l}\text {-Diagnóstico inicial de } \\
\text { estudiantes y docentes }\end{array}$ & $\begin{array}{l}\text {-Observación } \\
\text {-Comunicación oral }\end{array}$ & $\begin{array}{l}\text {-Cuestionario de } \\
\text { emociones } \\
\text {-Escala de estimación }\end{array}$ \\
\hline $\begin{array}{l}\text {-Desarrollo del constructo: } \\
\text {-Regulación emocional. }\end{array}$ & $\begin{array}{l}\text {-Observación } \\
\text {-Estrategias } \\
\text { emocionales }\end{array} \quad$ socio & $\begin{array}{l}\text {-Manejo de las emociones } \\
\text {-Rexión intrapersonal }\end{array}$ & \\
\hline
\end{tabular}




\section{-Praxis competencia social -Termómetro emocional -Habilidades cognitivas -Reflexión interpersonal}

\section{SOUTH FLORIDA \\ JOURNAL OF HEALTH}

\section{-Trabajo colectivo \\ -Conversatorios}

Tabla 2. Análisis de datos recolectados. Triangulación temporal.

Participantes

Antes

Durante

Después
Docente

-Carga cultural de autoritarismo e individualismo académico.

-Comunicación unidireccional -Educación vertical heredada.

-Académicos situados en su zona de confort, enfocados en el aspecto cognitivo.

-Conscientes de las emociones, pero no la aplican en el aula.
-Reconocimiento de la importancia de la existencialidad humana. -Sensibles en el de manejo de sus propias emociones mediante la reflexión autocrítica.

-Capaces de percibir, comprender y manejar sus emociones.

-Abiertos a la aplicación de estrategias emocionales a sus estudiantes, teniendo conciencia del valor que incide en la parte cognitiva.
-Organización de jornadas o talleres de educación socioemocional continua, con enfoque humanista $y$ dialéctico para la toma de decisiones en consenso mediante la auto regulación, que gestiona sobre los estados de ánimo y genera bienestar, al llevarlos a la praxis continúa en el proceso enseñanza y aprendizaje.

\begin{tabular}{ll}
\hline Estudiantes & -Nervios al no entender lo \\
que dice el profesor cuando & le pregunta algo y no sabe \\
que decir. & \\
-Temor al hablar inglés, por & creer no hacerlo bien. \\
& -Exponerse ante el grupo \\
& como malo y sentir pena. \\
& -Baja autoestima al no \\
& alcanzar los objetivos \\
& planteados y compararse \\
& con otros compañeros. \\
& - Se aíslan o desertan al \\
& intentarlo y no poder \\
& hacerlo mejor.
\end{tabular}

-Reflexión sobre su propia identidad.

-Mejora su auto estima y confianza en sí mismo.

-Toma conciencia de las emociones sobre los aspectos intelectual y actitudinal en la práctica educativa. -Sensibilización de la importancia de las emociones para el mejoramiento personal $y$ -Mejor conexión entre estudiantes y docentes logrando avances significativos en el proceso socio cognitivo mediante espacios de respeto por las emociones entre ambas partes, generando confianza y seguridad, basado en diálogos afectivos ideal para el aprendizaje del idioma. cognitivo. 


\section{REFERENCIAS}

[1] R. Bisquerra., «"Educación emocional y competencias básicas para la vida",» Revista de Investigación Educativa, vol. 21, nº 1, pp. 7-47, 2003.

[2] G. Moskowitz, Caring and Sharing in the Foreign Language Class. A Sourcebook on Humanistic Techniques, Boston: Newbury House Publishers, 1978.

[3] A. T. a. S. Meyer, Socialization of emotion regulation in the family, California: University of California, 2007.

[4] S. McMinn, "'Podcasting possibilities: Increasing time and motivation in the language learning classroom"," European Institute for E-Learning Forum, pp. 212-215, 2008.

[5] M. T. y. A. V. C. Bueno, «"La inteligencia emocional en alumnos de Magisterio: la percepción y comprensión de los sentimientos y las emociones",» Revista Interuniversitaria del Profesorado, vol. 19, no 54, pp. 169-194, 2005.

[6] C. Hué, Una experiencia de formación en competencias emocionales del profesorado universitario, Zaragoza: Universidad de Zaragoza, 2007.

[7] S. Krashen., Second Language Acquisition and Second Language Learning, Oxford: Pergamon press, 1981.

[8] J. L. Y. N. L. A. Peñalva, “"Competencias emocionales del alumnado de Magisterio: posibles implicaciones profesionales”,» Revista de Educación, n 362, pp. 690-712, 2013.

[9] E. Garcia, Formación del profesorado en educación emocional: Diseño, aplicación y evaluación.(trabajo doctoral), Barcelona: Universitat de Barcelona., 2011.

[10] C. P. y. W. F. F. Chamorro, «“Actitudes y emociones: pautas para el aprendizaje del,» Revista electrónica de conocimientos, saberes y prácticas, vol. 1, nº 1, pp. 72-80, 2018.

[11] P. García, La inteligencia emocional en el estudiante universitario de licenciatura durante el, México: Tecnológico de Monterrey., 2012.

[12] A. T. y. R. C. D. Tacca, «"Inteligencia emocional del docente y satisfacción académica del estudiante universitario",» Revista Scielo, vol. 14, n 1, 2020.

[13] L. Vygotsky, Psicología y pedagogía, Madrid: Akal S.A., 2004.

[14] A. Maslow, Motivación y personalidad, Madrid: Diaz de Santos, 2006.

[15] L. Barret, How emotions are made, Boston: Houghton Mifflin Harcourt, 2017.

[16] J. Elliot, El cambio educativo desde la investigación acción, Madrid: Morata, 2000.

[17] M. Santos, Hacer posible lo cotidiano. Teoría y práctica de la evaluación cualitativa de centros escolares, Madrid: Akal, 1990. 